\title{
A Study on the Relationship among Transformational Leadership, Organizational Identification and Voice Behavior
}

\author{
Dongbo Hu, Binbin Zhang, Meng Wang \\ Management School, Jinan University, Guangzhou, China \\ Email: zhangbzbz@126.com
}

Received 5 January 2015; accepted 25 February 2015; published 28 February 2015

Copyright (C) 2015 by authors and Scientific Research Publishing Inc.

This work is licensed under the Creative Commons Attribution International License (CC BY). http://creativecommons.org/licenses/by/4.0/

\section{(c) (7) Open Access}

\begin{abstract}
This paper investigates the relationship between transformational leadership and voice behavior under Chinese cultural background. It also examines the mediating effects of organizational identification in the relationship between transformational leadership and voice behavior. We use multiple linear regression analyses to validate our hypotheses. The results of the empirical study showed that: Transformational leadership has a significant positive influence on voice behavior; transformational leadership and its four dimensions have a significant positive influence on organizational identification; organizational identification has a significant positive influence on voice behavior. Organizational identification plays a part intermediary role between transformational leadership and voice behavior.
\end{abstract}

Keywords

Leadership Theory, Transformational Leadership, Organizational Identification, Voice Behavior

\section{Introduction}

More and more enterprises begin to focus on voice behavior. Chiabum (1998) proved voice behavior is beneficial to enhance organizational vitality and innovation. Scholars study voice behavior from two respects including individual factors and organizational situation factors [1].

Burns (1978) thinks transformational leaders have a high professional competence and accomplishment. They can stimulate their employees' enthusiasm and creativity, encouraging them to improve themselves [2]. Bass 
(1985) believes that the most important task for transformational leaders is to make employees understand the necessity to finish the job and let them realize the responsibility to bear. They also should motivate employees to pursuit high level of fame and pay attention to enhance the organization's overall performance to complete team target brilliantly [3].

LePine (2001) defines the voice behavior is a contributing and challenging initiative behavior, including constructive and change-oriented communication to improve the situation [4]. Ashforth (1989) believes that organizational identification can be viewed as a special case of social identity, which belongs to the category of group consciousness [5]. Weiling Xu (2002) thinks that organizational identification is a process of individual self-defined, while self-concept and organization belonging play a role [6]. Jun Wei (2004) believes organizational identification is a kind of subjective feelings which is produced in the individual's absorption of organizational factors [7].

As an important style of leadership, transformational leadership has an important influence on the formation of corporate culture; its impact and mechanism on employee voice behavior have not been deeply studied. In one organization, improving employees' organizational identification plays a very important role to promote employees' satisfaction and performance. In addition to meeting its own employee development, the relationship among transformational leadership, organizational identification and voice behavior is a question worth considering.

Rooted in the context of Chinese culture are not currently in-depth research, relevant empirical research is lacking. Voice behavior under Chinese cultural background has certain challenges and risks; it may touch the interests of certain colleagues or leadership causing tension in the relationship. Under the influence of Chinese traditional thought, the Chinese company's employees are more inclined to obey superiors' decisions or to remain silent while they face to practical problems. Therefore, it is very necessary to research the relationship between transformational leadership and voice behavior by using scale which is more in line with the actual situation of Chinese enterprises. Based on the above background, this paper adopts empirical methods to explore relationship among transformational leadership, organizational identification and voice behavior.

\section{Literature Review and Hypothesis}

Many scholars have confirmed that transformational leadership has a significantly positive correlation with organizational citizenship behavior, while voice behavior is one of employee extra role behaviors. We believe that transformational leadership can promote the voice behavior. Milliken (2003) finds that transformational leadership can inspire employees to pay beyond the expectations of efforts by establishing mutual trust, while voice behavior is one of the behaviors beyond the organizations' expectations to employees [8]. Piccolo verifies a significant relationship between transformational leadership and organizational citizenship behaviors. Long-zeng Wu (2011) finds that transformational leadership has a significant positive correlation with voice behavior through empirical research [9].

Based on the analysis above, this paper puts forward the following hypothesis:

H1: Transformational leadership has a significant positive correlation with voice behavior.

H1-1: Transformational leadership has a significant positive correlation with promotive voice behavior;

H1-1a: Virtue has a significant positive correlation with promotive voice behavior;

H1-1b: Incentive vision has a significant positive correlation with promotive voice behavior;

H1-1c: Personalized care has a significant positive correlation with promotive voice behavior;

H1-1d: Charisma has a significant positive correlation with promotive voice behavior;

H1-2: Transformational leadership has a significant positive correlation with prohibitive voice behavior;

H1-2a: Virtue has a significant positive correlation with prohibitive voice behavior;

H1-2b: Incentive vision has a significant positive correlation with prohibitive voice behavior;

H1-2c: Personalized care has a significant positive correlation with prohibitive voice behavior;

H1-2d: Charisma has a significant positive correlation with prohibitive voice behavior.

Organizational identification emphasizes the recognition of the organizations and further a sense of belonging. The stronger sense of belonging, the larger power internalization organization's goals, and the individual's work pay will be higher. Employee's pay includes two aspects: role behaviors and extra role behaviors. Riketta thinks that if employees have stronger organizational identification, they are more likely to exhibit extra role behaviors. Bell (2002) concluded if employees have stronger organizational identification, they are more likely to exhibit 
extra role behaviors to enhance customer perception of quality and service [10]. Ying Cai Zhai (2010) finds that all the three dimensions of organizational identification can motivate employees to produce extra role behaviors [11]. Lu Wang (2011) has found occupation identification and team identification have a positive correlation with voice behavior [12].

Based on the analysis above, this paper puts forward the following hypothesis:

H2: Organizational identification has a significant positive correlation with voice behavior;

H2-1: Organizational identification has a significant positive correlation with promotive voice behavior;

H2-2: Organizational identification has a significant positive correlation with prohibitive voice behavior.

We can find that transformational leadership has a positive impact on employees' behavior from other scholars' studies. Ashforth first puts forward the hypothesis that transformational leadership may impact the organizational identification. Epitropaki thinks organizational identification stems from the pursuit of higher goals, while the leader plays the role of incentives in the process. Kark (2003) believes transformational leadership enables to build mutual trust and respect between the leaders and employees, to establish the employees' organizational identification by transforming the leader's values and goals into self-pursuit [13]. XinYi Mao (2009) finds that transformational leadership has a significant correlation with organizational identification [14]. LiangDing Jia finds that transformational leadership has a significant positive impact on employee organizational commitment.

Based on the analysis above, this paper puts forward the following hypothesis:

H3: Transformational leadership has a significant positive correlation with organizational identification;

H3-1: Virtue has a significant positive correlation with organizational identification;

H3-2: Incentive vision has a significant positive correlation with organizational identification;

H3-3: Personalized care has a significant positive correlation with organizational identification;

H3-4: Charisma has a significant positive correlation with organizational identification.

Transformational leadership can greatly enhance the employees' organizational identification and responsibility. Organizational identification can stimulate employees' sense of responsibility and mission, believing them having the responsibility and ability to promote the development of the organization performing voice behavior more. Therefore, we hypothesize:

H4: Organizational identification plays an intermediary role between transformational leadership and voice behavior.

\section{Research Methodologies}

\subsection{Research Sample}

This research data mainly acquired through on-site questionnaire. In this survey, 300 questionnaires are distributed, 233 valid questionnaires are recovered, and the effective recovery rate is $77.7 \%$. The survey sample comes from different industry employees, including financial, manufacturing, retail, communications industry and so on, covering the areas of Guangdong Province, Anhui Province, Shandong Province and so on. The staff level distribution is as follows, general staff accounting for $27.9 \%$, first-line managers accounting for $55.8 \%$, middle managers accounting for $16.3 \%$. The ratio of male and female is 126:107. The sample education contains high school diploma and below accounting for $4.3 \%$, college diploma accounting for $24.5 \%$, bachelor degree accounting for $71.2 \%$. The staff age distribution is as follows, below 20 accounting for $14.2 \%, 20-30$ accounting for $40.8 \%$, above 30 accounting for $45 \%$. Working years, below 2 years accounting for 19.3\%, 2 - 5 years accounting for $25.3 \%, 5$ - 10 years accounting for $23.6 \%$, above 10 years accounting for $31.8 \%$.

\subsection{Measurement Tools}

In this study, we take the Likert Five Points Scale for all three scales, each question item are made of the positive points, that is, from " 1 - totally inconsistent" to " 5 -fully consistent". The questionnaire is divided into four parts: basic personal information, transformational leadership scale, voice behavior scale and organizational identification scale.

Taking the characteristics of Chinese-style management situations into account, the transformational leadership scale used is revised by Li Chao-ping according to Bass Multifactor Leadership Questionnaire scale, the scale includes 4 subscales, namely virtue, incentive vision, charisma, personalized care, respectively contain 8 , 
6,6 and 6 items. Internal consistency coefficients of the four dimensions are above 0.8 , indicating that the scale has good reliability. Using revised two-dimensional voice behavior scale by Liang and Farh, promotive and prohibitive voice behavior has 5 items respectively in original scale. In this study, the organizational identification scale used is a three-dimensional scale revised by Miller, the scale includes 3 subscales, namely cognitive, affective, evaluative, respectively contain 5, 4 and 3 items. The reliability of each scale is larger than 0.75 , which means that the research data obtained has good reliability.

\subsection{Procedure}

This paper tests the reliability of the questionnaire through reliability analysis, and uses correlation analysis method to analyze the correlation among transformational leadership, organizational identification and voice behavior, finally explores the linear relationship between variables by linear regression analysis.

\section{Data Analysis and Results}

\subsection{Correlation Analysis of Variables}

Using SPSS19.0 to analyze the correlation, as Table 1 shows, we can draw the following conclusions: 1) The correlation coefficient between transformational leadership and two dimensions of voice behavior (promotive, prohibitive) are $0.769,0.731,0.669$, namely there are a significant positive correlation in $\mathrm{P}<0.01$ level. H1, H1-1, H1-2 are verified; 2) The correlation coefficient between organizational identification and two dimensions of voice behavior (promotive, prohibitive) are $0.747,0.877,0.627$, namely there are a significant positive correlation in P < 0.01 level. H2, H2-1, H2-2 are verified; 3) The correlation coefficient between transformational leadership and organizational identification is $0.764, \mathrm{H} 3$ is verified.

\subsection{Regression Analysis}

\subsubsection{Regression Analysis between Transformational Leadership and Organizational Identification}

In regression analysis, we first use the four dimensions of transformational leadership as the independent variable and the three dimensions of organizational identification as dependent variables. From Table 2, we know that significant positive correlation exist between virtue and all the three dimensions of organizational identification in $\mathrm{P}<0.05$ level, and the regression coefficients are $0.181,0.116$, and 0.046 respectively. Therefore, H3-1 sets up. The regression coefficients between incentive vision and the three dimensions of organizational identification are $0.314,0.294,0.142$ respectively, it indicate that incentive vision can deepen employees' organizational identification. H3-2 is verified. We can also know that personalized care and charisma are in significant linear correlation with three dimensions of organizational identification. H3-3, H3-4 are verified.

Table 1. Correlations.

\begin{tabular}{|c|c|c|c|c|c|c|c|c|c|}
\hline & 1 & 2 & 3 & 4 & 5 & 6 & 7 & 8 & 9 \\
\hline 1 Virtue & 1 & & & & & & & & \\
\hline 2 Incentive vision & $0.460^{* *}$ & 1 & & & & & & & \\
\hline 3 Personalized care & $0.338^{* *}$ & & 1 & & & & & & \\
\hline 4 Charisma & $0.300^{* *}$ & & & 1 & & & & & \\
\hline 5 Transformational leadership & $0.795^{* *}$ & $0.718^{* *}$ & $0.651^{* *}$ & $0.620^{* *}$ & 1 & & & & \\
\hline 6 Organizational identification & $0.560^{* *}$ & $0.621^{* *}$ & $0.577^{* *}$ & $0.390^{* *}$ & $0.764^{* *}$ & 1 & & & \\
\hline 7 Promotive voice behavior & $0.534^{* *}$ & $0.584^{* *}$ & $0.601^{* *}$ & 0.139 & $0.731^{* *}$ & $0.877^{* *}$ & 1 & & \\
\hline 8 Prohibitive voice behavior & $0.467^{* *}$ & $0.589^{* *}$ & $0.564^{* *}$ & 0.093 & $0.669^{* *}$ & $0.627^{* *}$ & $0.533^{* *}$ & 1 & \\
\hline 9 Voice behavior & $0.601^{* *}$ & $0.605^{* *}$ & $0.615^{* *}$ & $0.331^{* *}$ & $0.769^{* *}$ & $0.747^{* *}$ & $0.635^{* *}$ & $0.855^{* *}$ & 1 \\
\hline
\end{tabular}

${ }^{* *}$ Correlation is significant at the 0.01 level (2-tailed). 


\subsubsection{Regression Analysis between Organizational Identification and Voice Behavior}

We use the organizational identification as the independent variable and the two dimensions of voice behavior as dependent variables. From Table 3, we know that organizational identification on promotive and prohibitive voice behavior has significant positive effects, the standardization regression coefficients are 0.889 and 0.116 , significant at 0.001 level. Therefore, H2-1 and H2-2 support.

\subsubsection{Regression Analysis between Transformational Leadership and Voice Behavior}

We use the four dimensions of transformational leadership as the independent variable and use the promotive and prohibitive voice behavior as dependent variables. As Table 4 shows, virtue, incentive vision, personalized care has significant correlation with promotive and prohibitive voice behavior. H1-1a, H1-1b, H1-1c, H1-2a, H1-2b, and H1-2c are verified. However, charisma has no significant correlation with promotive and prohibitive voice behavior, H1-1d and H1-2d don't set up.

\subsubsection{Testing the Intermediary Role of Organizational Identification}

In this study, we adopt Baron and Kenny (1986) method to test the mediating effect of organizational identification. We use transformational leadership as the independent variable and use the promotive and prohibitive voice behavior as dependent variables. The first step is to examine the effect of the four dimensions of transformational leadership on the promotive and prohibitive voice behavior, the second step is to examine the effect after considering the intermediary variable. As can be seen from Table 5, the effect of virtue on voice behavior changes from a significant correlation to no significant correlation after organizational identification is included in the regression equation; Correlation between the incentive vision, personalized care and voice behavior

Table 2. Regression analysis result between transformational leadership and organizational identification.

\begin{tabular}{cccc}
\hline Model 1 & Cognitive & Affective & Evaluative \\
\cline { 2 - 4 } & $\beta$ & $\beta$ & $0.046^{*}$ \\
Virtue & $0.181^{* * *}$ & $0.116^{* * *}$ & $0.142^{*}$ \\
Incentive vision & $0.314^{* * *}$ & $0.294^{* * *}$ & $0.077^{*}$ \\
Personalized care & $0.371^{* * *}$ & $0.193^{* * *}$ & $0.133^{*}$ \\
Charisma & $0.093^{*}$ & $0.115^{*}$ & \\
\hline
\end{tabular}

${ }^{*} \mathrm{p}<0.05 ; \stackrel{* *}{\mathrm{p}}<0.01 ;{ }^{* * *} \mathrm{p}<0.001$.

Table 3. Regression analysis result between organizational identification and voice behavior.

\begin{tabular}{ccc}
\hline \multirow{2}{*}{ Model 2} & Promotive voice behavior & Prohibitive voice behavior \\
\cline { 2 - 3 } & $\beta$ & $\beta$ \\
\hline Organizational identification & $0.889^{* * *}$ & $0.116^{* * *}$ \\
\hline
\end{tabular}

${ }^{*} \mathrm{p}<0.05 ;{ }^{* *} \mathrm{p}<0.01 ;{ }^{* * *} \mathrm{p}<0.001$.

Table 4. Regression analysis result between transformational leadership and voice behavior.

\begin{tabular}{ccc}
\hline & Promotive voice behavior & Prohibitive voice behavior \\
\cline { 2 - 3 } Model 3 & $\beta$ & $\beta$ \\
\hline Virtue & $0.188^{* * *}$ & $0.226^{* * *}$ \\
Incentive vision & $0.324^{* * *}$ & $0.289^{* * *}$ \\
Personalized care & $0.369^{* * *}$ & $0.283^{* * *}$ \\
Charisma & 0.097 & 0.014 \\
\hline
\end{tabular}

${ }^{*} \mathrm{p}<0.05 ;{ }^{* *} \mathrm{p}<0.01 ;{ }^{* * *} \mathrm{p}<0.001$. 
Table 5. Testing the intermediary role of organizational identification.

\begin{tabular}{ccccc}
\hline \multirow{2}{*}{ Model 4 } & \multicolumn{2}{c}{ Promotive voice behavior } & \multicolumn{2}{c}{ Prohibitive voice behavior } \\
\cline { 2 - 5 } & Step 1 & Step 2 & Step 1 & Step 2 \\
\hline Virtue & $0.188^{* * *}$ & 0.075 & $0.226^{* * *}$ & 0.02 \\
Incentive vision & $0.324^{* * *}$ & $0.085^{*}$ & $0.289^{* * *}$ & $0.276^{* * *}$ \\
Personalized care & $0.369^{* * *}$ & $0.155^{* * *}$ & $0.283^{* * *}$ & $0.272^{* * *}$ \\
Charisma & 0.097 & -0.003 & 0.014 & 0.009 \\
Organizational identification & & $0.751^{* * *}$ & & 0.041 \\
\hline
\end{tabular}

${ }^{*} \mathrm{p}<0.05 ;{ }^{* *} \mathrm{p}<0.01 ;{ }^{* * *} \mathrm{p}<0.001$.

significantly weaken. Therefore, we think that Organizational identification plays a part intermediary role between transformational leadership and voice behavior. H4 is partially verified.

\section{Conclusion and Suggestion}

\subsection{Conclusion}

Through empirical research, this paper examines the relationship among transformational leadership, organizational identification and voice behavior. The results of the empirical study showed that:

1) Transformational leadership has a significant positive influence on voice behavior, and the influence on promotive voice behavior is much stronger than on prohibitive voice behavior.

2) Transformational leadership and its four dimensions have a significant positive influence on organizational identification.

3) Organizational identification has a significant positive influence on both promotive voice behavior and prohibitive voice behavior.

4) Organizational identification plays an intermediary role between transformational leadership and voice behavior.

\subsection{Suggestion}

Based on the conclusions of this study, we propose the following management recommendations:

Enterprise leaders should treat promoting transformational leadership as a strategic goal. They must make employees understand the purpose and significance to implement transformational leadership. Specifically, managers should enhance leadership skills, and constantly enrich and improve the theoretical knowledge, to impress and influence people through personal charm. They should convince employees to obey the leaders, being willing to follow the leadership together to complete organizational goals. It requires managers to be able to describe the organization's future direction and strategic planning, stimulate employees' enthusiasm and spirit clearly, motivating staffs to complete the ambitious goals of the organization and struggle constantly. Managers should also observe and analyze the characteristics of subordinates, using individualized leadership to provide staff with customized care and to enhance the psychological sense of belonging to the organization staff.

In the context of Chinese culture, leaders should pay attention to morality and character self-cultivation using moral power to influence and appeal of employees. Organizations should improve employees’ organizational identification. This research shows that employees who have a stronger organizational identification are more likely to exhibit higher levels of voice behavior. If leaders want to hear the real voices of employees, they must let employees have a right to speak, to let them participate in making decisions. Only employees being involved in decision-making, they can identify problems in the implementation of decisions and suggest improvements. So to build a sound mechanism is the first step to ensure employees voice behavior.

For suggestions and behavior of employees, if there is no effective feedback mechanism to ensure the sustainability of voice behavior, then the employee voice behavior in the organization will be short-lived. Managers must first do humbly take advice and listen to the impolite sound. The managers should listen carefully and timely responses of different opinions and ideas of employees, inspiring employee enthusiasm; secondly, man- 
agers should respect for employees, even if the unreasonable proposal should be given full respect. Leaders' attitude will largely determine those who offer advice and suggestions.

In the specific business management operations, enterprises should actively expand the staff suggestions channels, through the game, seminars, presentations, surveys, e-mail suggestions to encourage staff in various ways; through corporate Web site, OA office systems, weekly magazine, employee suggestions should be published way out and reward high-quality advice for good results; enterprise should establish review panels and review the recommendations regularly; various departments should establish a common processes to implement voice behavior, establishing a closed-loop system and promoting the high feasibility suggestions into productivity.

\section{References}

[1] Janssen, O., De Vries, T. and Cozijnzen, A.J. (1998) Voicing by Adapting and Innovating Employees: An Empirical Study on How Personality and Environment Interact to Affect Voice Behavior. Human Relations, 51, 945-967. http://dx.doi.org/10.1177/001872679805100705

[2] Burus, J.M. (1978) Leadership. Harper and Row Press, New York.

[3] Bass, B.M. (1985) Leadership and Performance beyond Expectations. Free Press, New York, 3-342.

[4] Lepine, J.A. and Vandyne, L. (2001) Voice and Cooperative Behavior as Contrasting Forms of Contextual Performance: Evidence of Differential Relationships with Big Five Personality Characteristics and Cognitive Ability. Journal of Applied Psychology, 86, 326-336. http://dx.doi.org/10.1037/0021-9010.86.2.326

[5] Ashforth, B.E. and Mael, F. (1989) Social Identity Theory and the Organization. Academy of Management Review, 14, 20-39.

[6] Xu, W.L. and Zheng, B.X. (2002) Organizational Identification: A Preliminary Exploration of the theory and the Nature. Analysis of ZhongShan Management Review, 11, 45-64.

[7] Wei, J., Chen, Z.Y. and Zhang, M. (2007) The Basic Theory, Measurement and Related Variables of Organizational Identification. Advances in Psychological Science, 26, 948-955.

[8] Milliken, F.J., Morrison, E.W. and Hewlin, P.F. (2003) An Exploratory Study of Employee Silence: Issues That Employees Don’t Communicate Upward and Why. Journal of Management Studies, 40, 1453-1476. http://dx.doi.org/10.1111/1467-6486.00387

[9] Wu, L.Z., Cao, K.P., Chen, Y.Y., et al. (2011) A Study on Transformational Leadership Behavior Impact on Employee Voice Behavior. Journal of Management, 8, 61-80.

[10] Bell, S.J. and Menguc, B. (2002) The Employee-Organization Relationship, Organizational Citizenship Behaviors, and Superior Service Quality. Journal of Retailing, 78, 131-146. http://dx.doi.org/10.1016/S0022-4359(02)00069-6

[11] Zhai, Y.C. (2010) The Core Employee Organizational Identification. An Empirical Study of the Impact on Organizational Citizenship Behavior of Soochow University.

[12] Wang, L. and Gao, P. (2011) An Empirical Study on Occupation Identity, Team Identification Effect on Employee Voice Behavior. Practice and Understanding of Math., 41, 129-137.

[13] Kark, R., Shamir, B. and Chen, G. (2003) The Two Faces of Transformational Leadership: Empowerment and Dependency. Journal of Applied Psychology, 88, 246-255. http://dx.doi.org/10.1037/0021-9010.88.2.246

[14] Mao, M.X. and Long, L.R. (2009) Transformational Leadership and Employees on Organizational Change Recognition on the Research of the Relationship between Shared. Journal of Management, 6, 595-600. 
Scientific Research Publishing (SCIRP) is one of the largest Open Access journal publishers. It is currently publishing more than 200 open access, online, peer-reviewed journals covering a wide range of academic disciplines. SCIRP serves the worldwide academic communities and contributes to the progress and application of science with its publication.

Other selected journals from SCIRP are listed as below. Submit your manuscript to us via either submit@scirp.org or Online Submission Portal.
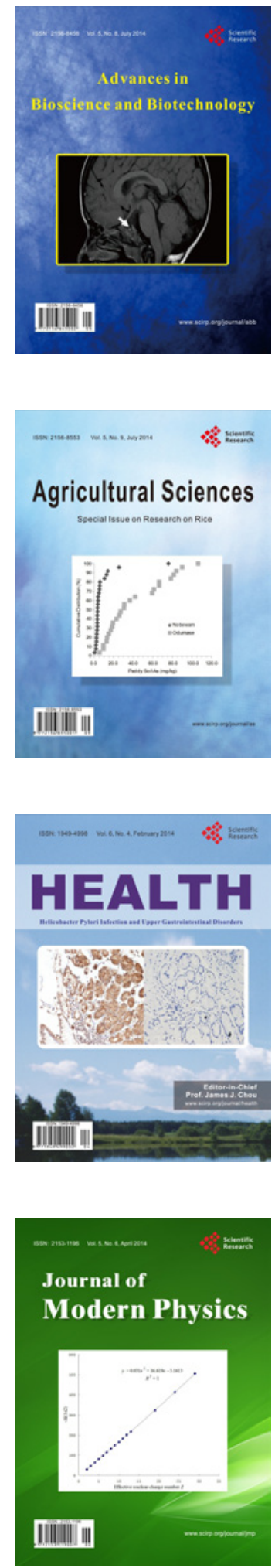
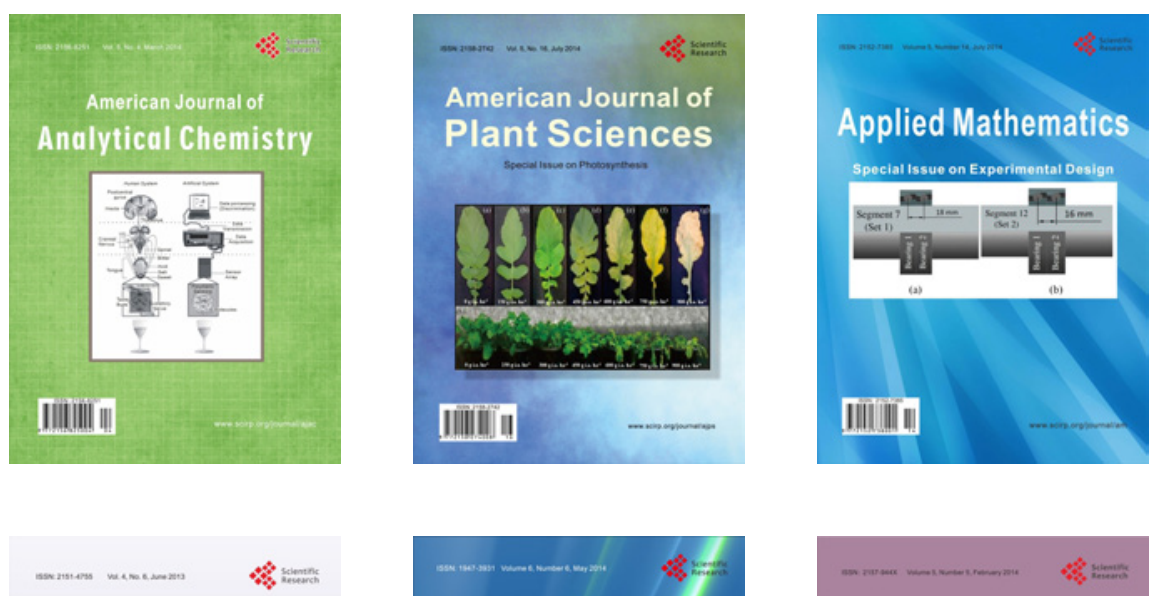

Creative Education
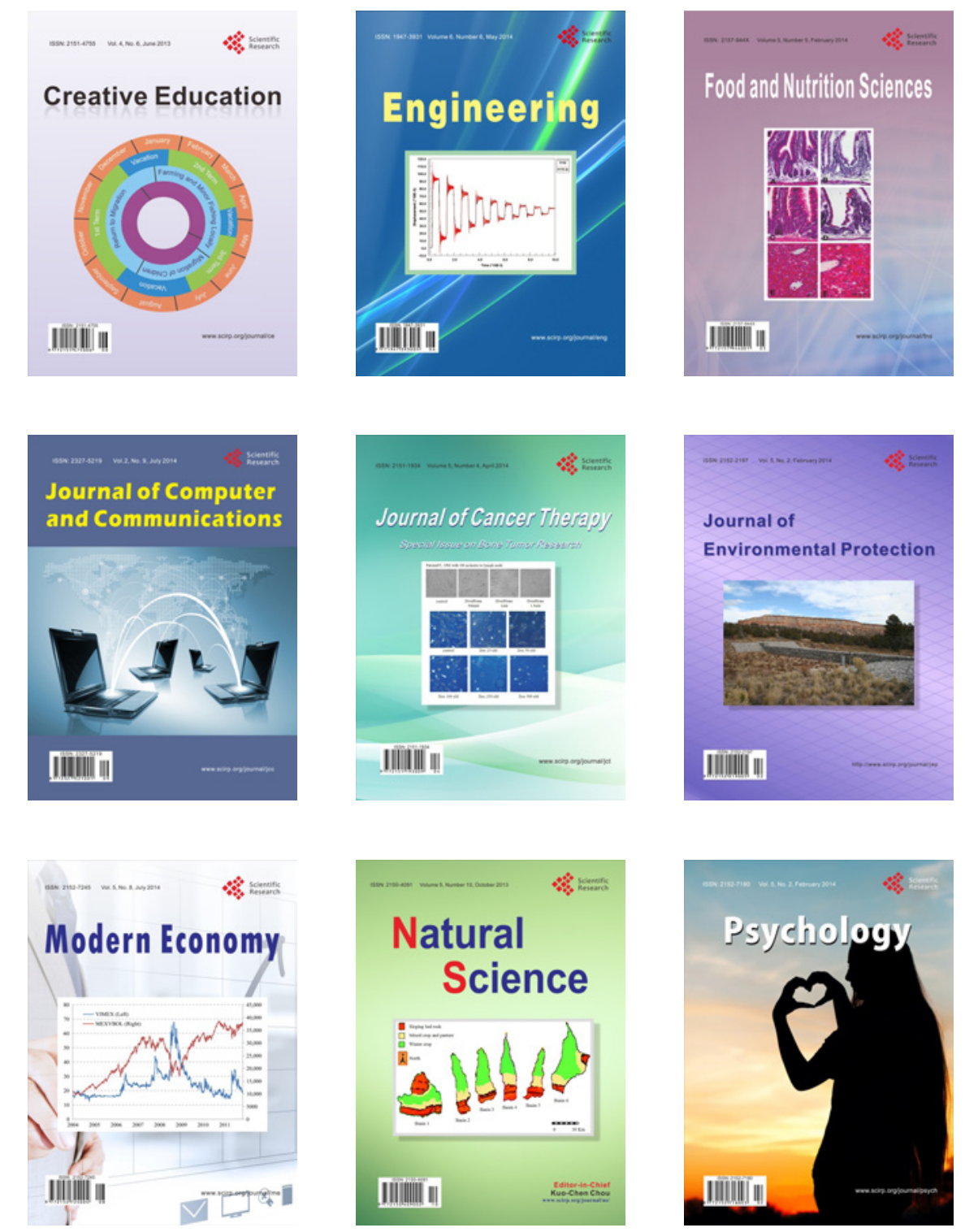